\title{
Efectos de un programa de intervención en la motricidad gruesa: estudio con niños de 5 a 7 años.
}

Effects of an intervention program in gross motor skills: study with children 5 to 7 years.

Julio Alfonso Mocha Bonilla ${ }^{1}$, Patricio Gustavo Ortiz Ortiz ${ }^{2}$, Esmeralda Giovanna Zapata ${ }^{3}$, Andrea Vanessa Cárdenas Zúñiga ${ }^{4}$

Recibido: 08-12-2017 / Revisado: 09-02-2018 Aceptado: 11-03-2018/ Publicado: 01-04-2018

\begin{abstract}
.
DOI: https://doi.org/10.33262/cienciadigital.v2i2.73

The teachers during the teaching - learning process of the Physical Education classes carry out the practice of physical and recreational activities seeking to generate this way the motor development in the children, the gross motor in the infantile stage of great importance because through it The skills and abilities to execute the body movements known as motor habits are strengthened. The research presents an intervention program based on recreational activities developed during six months in the physical education classes for which it was proposed to evaluate the gross motor skills of the first and second year of general basic education, the data were evaluated by means of The application of the MABC-2 test, by means of which the levels can be known, refers to gross motor skills. A total of 50 male children were worked on, which were the first and second years of Basic General Education, the five and seven years respectively. The results found significant evidence for the intervention of the
\end{abstract}

\footnotetext{
${ }^{1}$ Universidad Técnica de Ambato, Facultad de Ciencias Humanas y de la Educación, Carrera de Pedagogía de la Actividad Física y Deporte, Ambato, Ecuador, ja.mocha@uta.edu.ec

${ }^{2}$ Universidad Técnica de Ambato, Facultad de Ciencias Humanas y de la Educación, Ambato, Ecuador patriciogortizo@uta.edu.ec

${ }^{3}$ Universidad Técnica de Ambato, Facultad de Ciencias Humanas y de la Educación, Ambato Ecuador, eg.zapata@uta.edu.ec

${ }^{4}$ Universidad Técnica de Ambato/Facultad de Ciencias Humanas y de la Educación/Carrera de Cultura Física Ambato, Ecuador, acardenas4125@uta.edu.ec
} 
program in seven of the eight variables studied, with which it can be mentioned, instead, the program is called tuning in relation to the balance on one leg.

Keywords: Movement, Gross Motor, Motor Development, Physical Activity.

\section{Resumen.}

Los docentes, durante el proceso de enseñanza - aprendizaje de las clases de Educación Física, realizan la práctica de actividades físicas y recreativas con la finalidad de generar el desarrollo motor en los niños. La motricidad gruesa, en la etapa infantil, es de gran importancia, pues por medio de ella se afianzan las habilidades y destrezas para ejecutar los movimientos corporales más conocidos como hábitos motrices. La investigación presenta un programa de intervención basado en actividades recreativas desarrolladas durante seis meses en las clases de educación física, para lo cual, se propuso como objetivo evaluar la motricidad gruesa de los niños de primero y segundo año de educación general básica, los datos fueron evaluados mediante la aplicación de test MABC-2 que permite conocer los niveles referentes a la motricidad gruesa; se trabajó con un total de 50 niños varones, los mismos que corresponden al primero y segundo año de Educación General Básica; las edades estuvieron comprendidas entre los cincos y siete años respectivamente. Los resultados encontrados muestran evidencias significativas tras la intervención del programa en siete de las ocho variables estudiadas, con lo cual se puede mencionar que nuestra intervención produjo mejoras significativas en la motricidad gruesa de los sujetos; sin embargo, se debe afianzar el programa en lo referente al equilibrio sobre una pierna.

Palabras Claves: movimiento, motricidad gruesa, desarrollo motor, actividad física.

\section{Introducción.}

Andradas (2015) menciona que ser físicamente activo durante la niñez y adolescencia no sólo es primordial para la salud integral, más bien, es importante para lograr un buen estilo de vida. La motricidad gruesa es la capacidad y habilidad que posee el cuerpo para ejecutar diversos movimientos, una de las premisas que hay que tener presente es la creación de hábitos motrices que, en palabras de Caballero (citado en Molina, 2017), deben ser desarrollados mediante una correcta organización y planificación; las prácticas de Educación Física deben enfocarse hacia la mejora de la coordinación motriz durante la 
etapa del desarrollo infantil, para proporcionar a los infantes actividades estabilizadoras, lúdicas y psicomotrices.

La actividad física, realizada diariamente desde edades tempranas, mejora la salud general (Oblitas, 2006; Arruza et al., 2008; Carrillo, 2017), además, desarrolla una adecuada forma física en la población infantil y adolescente, aportando beneficios físicos y bienestar emocional, a corto y largo plazo. Otro aspecto fundamental en la adaptación motriz es la realización de actividades físicas espontáneas, es decir, realizadas de forma voluntaria, los cuales proveen beneficios multifactoriales con efectos en el sistema inmune (González, 2018). La actividad física en la infancia permite que los niños se sientan competentes realizando sus habilidades físicas, y es probable que en su edad adulta sean más activos y participativos, además, el juego contribuye en el desarrollo físico, cognitivo, socioemocional y del lenguaje (Loza, 2017), ya que se considerada como un aliado del aprendizaje porque a través de él, el niño ensaya, imagina y crea. Es una oportunidad para identificar sus potencialidades y limitaciones, por ende, las instituciones educativas, conjuntamente con los profesionales de la educación física, son los encargados de brindar espacios lúdicos y recreativos para fomentar el desarrollo motriz, generar nuevos procedimientos que ayuden a los infantes a desarrollar diferentes actividades a través del juego, pues la etapa infantil es primordial para que un niño desarrolle todas sus habilidades. Para la mayoría de los niños las clases de Educación Física son el momento propicio para realizar la práctica de actividades físicas (Martínez, 2017), por consiguiente, la Educación Física está considerada como un área que permite promocionar a la actividad física de forma directa e indirecta (Fernández Rivas, 2017); sin embargo, en la Unidad Educativa "Totoras" de la ciudad de Ambato se pudo observar que no se imparten estrategias didácticas (Ferrandis, 2017) para el fortalecimiento de la motricidad gruesa. Existe un grupo de niños que cursan el primero y segundo año de Educación General Básica, en los cuales se nota dificultades en la ejecución de las actividades motrices durante las prácticas de educación física, los problemas de coordinación, lateralidad y equilibrio generan preocupación en autoridades, docentes y educadores físicos que trabajan en los años superiores, pues los niños no poseen un desarrollo motriz idóneo para la edad respectiva, generando tropiezos en el proceso de enseñanza - aprendizaje.

\section{Desarrollo.}

\subsection{Revisión de la literatura.}

Con el pasar de los años se han probado varios instrumentos de medición para evaluar la motricidad, se ha utilizado el test de eficiencia manual, el cual permite conocer las diferencias que existen entre el brazo dominante y el no dominante (Márquez, 1998). La motricidad y la enseñanza son saberes que se imparten en el aula y en la práctica deportiva, 
por lo tanto, se define a la motricidad como un saber durante el proceso de enseñanza, es decir, las categorías cuerpo-motricidad son un eje de la enseñanza (Zuluaga, 2015). La motricidad humana es una experiencia educativa, un estudio que se fundamenta mediante planificaciones de Educación Física utilizando un conjunto de situaciones de aprendizaje basado en ejercicios de autonomía y construcción de conocimiento (Da Silva Carmo, 2015).

En relación a los instrumentos aplicados para conocer la motricidad gruesa en infantes existen varias alternativas las cuales se describen a continuación: el test TGMD-2, que permite obtener resultados confiables y válidos al ser aplicados en niños de cinco a diez años, utilizando el coeficiente de correlación y el método BLAND-ALTMAN (CanoCappellacciI, 2015), al aplicar la escala de desarrollo motor de ALBERTA AIMS, los resultados mostraron que el $63.6 \%$ de los niños presentan retraso en su desarrollo motor, siendo la causa principal la desnutrición (León, 2015); por otra parte, el test de TGMD-2 permite evaluar la motricidad (Cid, 2015). En cuanto a los programas de intervención motriz para conocer el nivel de motricidad gruesa de los niños, los resultados han permitido observar el nivel de motricidad promedio, mientras que las sesiones aplicadas a los niños evidencia mejoras significativas en el desarrollo de sus habilidades (Vallejos, 2016). Además se aplicó el test TGMD-2 y el índice de masa corporal para evaluar el desarrollo de la motricidad gruesa y la nutrición en niños, los resultados del test lo obtuvieron los niños del nivel de transición I, y en el estado nutricional, los dos grupos alcanzaron valores similares, concluyendo que existe obesidad en los niños (Santana, 2016).

La psicomotricidad es el eje de todo desarrollo evolutivo, el aprendizaje del lenguaje en los niños, debiendo ser desarrollada mediante movimientos y juegos, la aplicación de la Prueba de Desarrollo Battelle, como un instrumento del lenguaje comprensivo y expresivo, así como la motricidad gruesa y fina (Rodríguez, 2017). El test Motor SportComp permite que los docentes de educación física evalúen la coordinación motriz de sus estudiantes, su aplicación es sencilla y ayuda a detectar los problemas motrices, los resultados se analizan en base a la coordinación motriz global (Pérez, 2017).

Al aplicar juegos durante la infancia se observa el fortalecimiento de las habilidades motrices básicas durante la evaluación de los movimientos corporales en niños, tras la aplicación de una ficha de observación, basada en una guía didáctica de actividades lúdicas para la mejora de la motricidad gruesa, se concluye que el juego es una herramienta primordial en la etapa infantil y debe ser practicado de forma habitual (Bayas, 2017).

Por otra parte, el test de MABC-2, ayuda a identificar la precisión de lanzamiento y el rendimiento de captura en los niños, se concluye que una buena precisión de lanzamiento permite un buen rendimiento de captura de los objetos (Dirksen, 2016). De la misma manera el MABC 2 permite medir la destreza de puntería y atrape (Bravo, 2017). 
En definitiva, la batería MABC-2 es uno de los más reconocidos para la detección de problemas de coordinación motriz (Pérez, 2017), es decir, es una batería integral que identifica y evalúa el desarrollo motor en los niños (Henderson et al., citado en Hollund, 2018). La competencia motriz se debe evaluar usando la Batería del movimiento para niños, cuya segunda edición se la conoce como (MABC-2), una evaluación estandarizada de la habilidad motora fina y gruesa (Sumner, 2018).

En definitiva, la Batería de Evaluación del Movimiento para niños (MABC-2) es uno de los test más prestigiosos, y utilizados internacionalmente, para detectar los Trastornos del desarrollo de la coordinación en los niños de entre 4 y 16 años de edad (Henderson, 2016), por lo tanto, luego de revisar la literatura hemos definido a la prueba MABC-2 como la más apropiada para nuestro estudio.

\subsection{Metodología.}

\section{Sujetos.}

En el programa de intervención participaron 50 niños varones, los mismos que corresponden al primero y segundo año de Educación General Básica, cuyas edades estuvieron comprendidas entre los cincos y siete años, respectivamente, y poseen una condición socioeconómica de clase media.

\section{Protocolos.}

En primera instancia se ejecutó la revisión de la literatura sobre el objetivo a cumplir, posteriormente se procede con el trabajo de campo, aplicando la intervención antes y después de las actividades físico recreativas, pues una intervención permite una evaluación diagnóstica y el futuro diseño de planes de intervención adecuados (Otalvaro, 2016). Nuestro programa de intervención fue establecido mediante juegos lúdicos, los cuales se desarrollaron mediante actividades recreativas con juegos asociados a la edad de los participantes durante las clases de educación física, desarrolladas durante 6 meses, aplicando una planificación inductiva-deductiva; una vez conocida la finalidad del estudio se tomaron los datos de cada uno de los cincuenta participantes, una medición antes de iniciar la intervención, y posteriormente luego de los seis meses de trabajo.

\section{Procedimiento.}

Con los participantes se procedió a la aplicación del test, la participación de los niños fue totalmente voluntaria (González, 2017) se solicitó consentimiento de los padres de familia 
por la edad que presentan los participantes. En primera instancia se socializó que el método no tiene carácter invasivo, pues la metodología aplicada no afecta a los niños participantes, y es de gran ayuda para conocer cómo se encuentra la motricidad gruesa de los sujetos de estudio, ya que existe una relación entre desarrollo motor y actividad física (Weisstaub, 2017); todo el proceso se cumplió con los requisitos previstos en el pretest y se aplicó durante las clases de educación física en el mes de enero, durante el periodo escolar del año lectivo 2016-2017, posterior a los seis meses se procedió a aplicar el post test y evaluar la motricidad gruesa, de esta manera coincidimos con Benjumea (2017). La coordinación motriz en infantes es un requerimiento para la educación física, permite conocer el nivel de motricidad en niños, así como la coordinación de objetos a través de actividades continuas como carreras, saltos y giros.

\section{Instrumentos.}

El test de MABC-2 concordando con (Bravo, 2017) permite evaluar el desarrollo motor de los estudiantes. Durante la aplicación del test, los estudiantes realizaron en primera instancia la prueba de destreza manual la misma que contiene tres ejercicios: introducir monedas, enhebrar cuentas y dibujar el trazado; posteriormente se aplicó la prueba de puntería y atrape, la cual contiene dos ejercicios: atrapar el saquito y lanzar el saquito a una diana; para finalizar el test se administró la prueba de equilibrio, la misma que está conformada por tres ejercicios: equilibrio sobre una pierna, andar de puntillas y saltar sobre alfombrillas. Antes de la aplicación del test se les explicó a todos los niños en qué consiste el test y cuál es la finalidad del estudio. La información fue procesada utilizando el software estadístico IBM SPSS, versión 20.0 para comparar los resultados del antes y después de la intervención del programa de actividad física como lo usó Pumar Vidal (2015), para lo cual se utilizó el estadístico de correlación de los rasgos de Wilconxon con su correspondiente valor de p. aceptado de $<0,05$ para cada caso. Las variables analizadas en el estudio fueron: introducir monedas, enhebrar cuentas, dibujar el trazado, atrapar el saquito, lanzar el saquito a una diana, equilibrio sobre una pierna, andar de puntillas y saltar sobre alfombrillas.

\section{Resultados.}

En la tabla 1. Se puede apreciar el análisis del PRE -TEST DE MABC-2 aplicado a los estudiantes de primero y segundo año de Educación General Básica de la Unidad Educativa "Totoras". 
Tabla 1. Análisis del pre test en los niños de cinco y siete años.

\begin{tabular}{lccccc}
\hline \multirow{2}{*}{ Destrezas: } & \multicolumn{5}{c}{ PUNTUACIÓN } \\
\cline { 2 - 5 } & $\mathbf{1}$ & $\mathbf{2}$ & $\mathbf{3}$ & $\mathbf{4}$ & \multirow{2}{*}{ TOTAL } \\
\cline { 2 - 5 } & Mala & Regular & Buena & Excelente & \\
\hline Atrapar el saquito & 0 & 15 & 25 & 10 & 50 \\
Lanzar el saquito a una & 20 & 15 & 10 & 5 & 50 \\
diana & 19 & 14 & 12 & 5 & 50 \\
Introducir monedas & 13 & 17 & 20 & 0 & 50 \\
Enhebrar cuentas & 10 & 15 & 20 & 5 & 50 \\
Dibujar el trazado & 7 & 11 & 22 & 10 & 50 \\
Equilibrio sobre una pierna & 18 & 16 & 16 & 0 & 50 \\
Andar de puntillas & 17 & 13 & 12 & 8 & 50 \\
Saltar sobre alfombrillas & & & & & 5 \\
\hline
\end{tabular}

Elaborado por: Grupo de Investigación.

Figura 1. Resultados de la aplicación del pre test.

\section{Resultado del pre test de MABC-2}

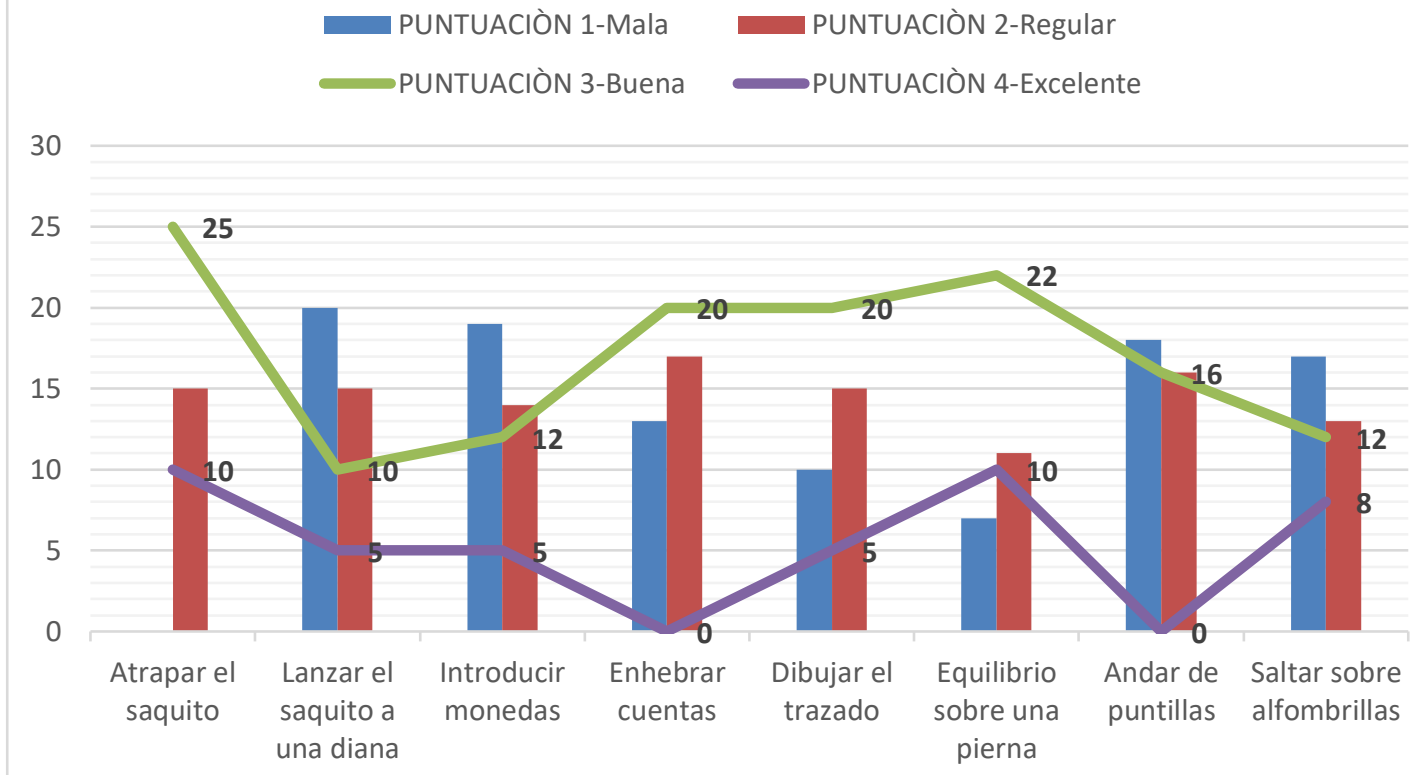

Elaborado por: Grupo de Investigación. 
En la figura 1. Se puede observar la aplicación del pre test; en la prueba de atrapar el saquito, de los 50 niños, 15 sujetos alcanzaron una puntuación regular, 25 sujetos alcanzaron una puntuación buena y 10 niños alcanzaron una puntuación excelente. En la prueba de lanzar el saquito a una diana 20 sujetos alcanzaron una puntuación mala, 15 sujetos alcanzaron la puntuación regular, 10 sujetos alcanzaron la puntuación buena y 5 sujetos alcanzaron la puntuación excelente.

En la prueba de introducir monedas 19 sujetos alcanzaron una puntuación mala, 14 sujetos alcanzaron la puntuación regular, 12 sujetos alcanzaron la puntuación buena y 5 sujetos alcanzaron la puntuación excelente. En la prueba de enhebrar cuentas 13 sujetos alcanzaron una puntuación mala, 17 sujetos alcanzaron la puntuación regular, 20 sujetos alcanzaron una puntuación buena y 0 sujetos alcanzaron la puntuación excelente.

En la prueba de dibujar el trazado 10 sujetos alcanzaron una puntuación mala, 15 sujetos alcanzaron la puntuación regular, 20 sujetos alcanzaron la puntuación buena y 5 sujetos alcanzaron la puntuación excelente. En la prueba de equilibrio sobre una pierna 7 sujetos alcanzaron una puntuación mala, 11 sujetos alcanzaron la puntuación regular, 22 sujetos alcanzaron la puntuación buena y 10 sujetos alcanzaron la puntuación excelente.

En la prueba de andar de puntillas 18 sujetos alcanzaron una puntuación mala, 16 sujetos alcanzaron la puntuación regular, 16 sujetos alcanzaron la puntuación buena y 0 sujetos alcanzaron la puntuación excelente.

Finalmente, en la prueba de saltar sobre alfombrillas 17 sujetos alcanzaron una puntuación mala, 13 sujetos alcanzaron la puntuación regular, 12 sujetos alcanzaron la puntuación buena y 8 sujetos alcanzaron la puntuación excelente.

En la tabla 2. Se puede observar el análisis del POS-TEST DE MABC-2 aplicado a los estudiantes de primero y segundo año de educación básica de la Unidad Educativa "Totoras".

Tabla 2. Análisis del post testen los niños de cinco y siete años.

\begin{tabular}{lccccc}
\hline \multirow{2}{*}{ Destrezas: } & \multicolumn{5}{c}{ PUNTUACIÓN } \\
\cline { 2 - 5 } & $\mathbf{1}$ & $\mathbf{2}$ & $\mathbf{3}$ & $\mathbf{4}$ & \multirow{2}{*}{ TOTAL } \\
\cline { 2 - 5 } & Mala & Regular & Buena & Excelente & \\
\hline Atrapar el saquito & 0 & 10 & 16 & 24 & 50 \\
Lanzar el saquito a una & 10 & 10 & 12 & 18 & 50 \\
diana & 15 & 10 & 9 & 16 & 50 \\
Introducir monedas & 7 & 17 & 16 & 10 & 50 \\
Enhebrar cuentas & & & & & \\
\hline
\end{tabular}


Dibujar el trazado 8 10 14 18

Equilibrio sobre una pierna 7

Andar de puntillas 10

14

13

16

Saltar sobre alfombrillas

9

13

12

15

50

13

12

16

50

Elaborado por: Grupo de Investigación.

Figura 2. Resultados de la aplicación del post test.

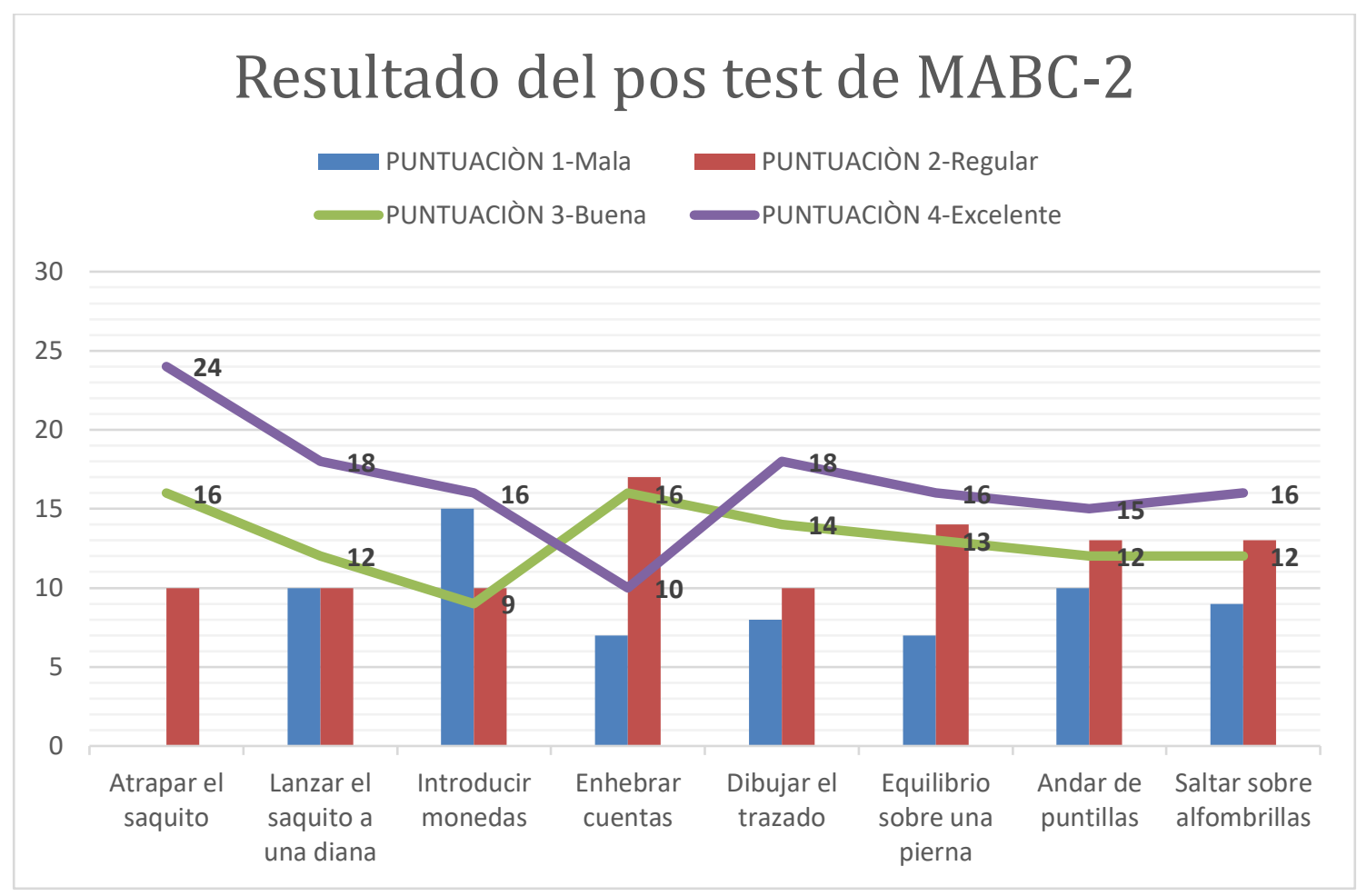

Elaborado por: Grupo de Investigación.

En la figura 2. Se puede analizar las diversas pruebas tras la aplicación del postest; en la prueba de atrapar el saquito de los 50 niños se puede mencionar que 10 sujetos alcanzaron una puntuación regular, 16 sujetos alcanzaron una puntuación buena y 24 niños alcanzaron una puntuación excelente.

En la prueba de lanzar el saquito a una diana 10 sujetos alcanzaron una puntuación mala, 10 sujetos alcanzaron la puntuación regular, 12 sujetos alcanzaron la puntuación buena y 18 sujetos alcanzaron la puntuación excelente. 
En la prueba de introducir monedas 15 sujetos alcanzaron una puntuación mala, 10 sujetos alcanzaron la puntuación regular, 9 sujetos alcanzaron la puntuación buena y 16 sujetos alcanzaron la puntuación excelente.

En la prueba de enhebrar cuentas 7 sujetos alcanzaron una puntuación mala, 17 sujetos alcanzaron la puntuación regular, 16 sujetos alcanzaron la puntuación buena y 10 sujetos alcanzaron la puntuación excelente. En la prueba de dibujar el trazado 8 sujetos alcanzaron una puntuación mala, 10 sujetos alcanzaron la puntuación regular, 14 sujetos alcanzaron la puntuación buena y 18 sujetos alcanzaron la puntuación excelente.

En la prueba de equilibrio sobre una pierna 7 sujetos alcanzaron una puntuación mala, 14 sujetos alcanzaron la puntuación regular, 13 sujetos alcanzaron la puntuación buena y 16 sujetos alcanzaron la puntuación excelente.

En la prueba de andar de puntillas 10 sujetos alcanzaron una puntuación mala, 13 sujetos alcanzaron la puntuación regular, 12 sujetos alcanzaron la puntuación buena y 15 sujetos alcanzaron la puntuación excelente. Finalmente, en la prueba de saltar sobre alfombrillas 9 sujetos alcanzaron una puntuación mala, 13 sujetos alcanzaron la puntuación regular, 12 sujetos alcanzaron la puntuación buena y 16 sujetos alcanzaron la puntuación excelente.

3.1 Estadístico de comprobación de la hipótesis.

Tabla No 3. Estadística.

\begin{tabular}{lccccc}
\hline \multicolumn{5}{c}{ Estadísticos de contraste $^{\mathbf{a}}$} \\
& $\begin{array}{c}\text { Atrapar el } \\
\text { saquito - } \\
\text { Atrapar el } \\
\text { saquito }\end{array}$ & $\begin{array}{c}\text { Laquito a una } \\
\text { diana - Lanzar } \\
\text { el saquito a } \\
\text { una diana }\end{array}$ & $\begin{array}{c}\text { Introducir } \\
\text { monedas - } \\
\text { Introducir } \\
\text { monedas }\end{array}$ & $\begin{array}{c}\text { Enhebrar } \\
\text { cuentas - } \\
\text { Enhebrar } \\
\text { cuentas }\end{array}$ & $\begin{array}{c}\text { Dibujar el } \\
\text { trazado - } \\
\text { Dibujar el } \\
\text { trazado }\end{array}$ \\
$\begin{array}{l}\text { Z } \\
\begin{array}{l}\text { Sig. asintót. } \\
\text { (bilateral) }\end{array}\end{array}$ & $-4,359^{\mathrm{b}}$ & $-5,708^{\mathrm{b}}$ & $-4,796^{\mathrm{b}}$ & $-4,690^{\mathrm{b}}$ & $-4,690^{\mathrm{b}}$ \\
\hline
\end{tabular}

Elaborado por: Grupo de Investigación.

Tabla No 4. Estadística de contrate. 


\begin{tabular}{cccc}
\hline \multicolumn{3}{c}{ Estadísticos de contraste $^{\mathrm{a}}$} & \\
\hline & $\begin{array}{c}\text { Equilibrio } \\
\text { sobre una } \\
\text { pierna - } \\
\text { Equilibrio } \\
\text { sobre una } \\
\text { pierna }\end{array}$ & $\begin{array}{c}\text { Andar de } \\
\text { puntillas - } \\
\text { Andar de } \\
\text { puntillas }\end{array}$ & $\begin{array}{c}\text { Saltar sobre } \\
\text { alfombrillas - } \\
\text { Saltar sobre } \\
\text { alfombrillas }\end{array}$ \\
$\begin{array}{c}\text { Sig. asintót. } \\
\text { (bilateral) }\end{array}$ & 0,317 & $-5,831^{\mathrm{b}}$ & $-4,899^{\mathrm{b}}$ \\
\hline
\end{tabular}

Elaborado por: Grupo de Investigación.

a. Prueba de los rangos con signo de Wilcoxon

b. Basado en los rangos negativos.

El estadístico de contraste muestra que, de los ocho valores de las variables de estudio, el correspondiente al equilibrio sobre una pierna cuyo p-valor "sig. Asintót. $=0.317>0.05$ muestra que no existe evidencia significativa de mejora en este aspecto entre la pre y pos intervención, mientras que los demás valores obtuvieron diferencias significativas luego del programa de intervención para las variables de: Atrapar el saquito, lanzar el saquito a una diana, introducir monedas, enhebrar cuentas, dibujar el trazado, andar de puntillas y saltar sobre alfombrillas cuyo p-valor "sig. Asintót. es $0.00<0.05$.

\section{Conclusiones.}

- La motricidad gruesa de los niños de estudio al momento de la aplicación del pre test se encontró con un nivel moderado, deduciendo así que cada una de las destrezas observadas deben ser desarrollas mediante programas de intervención para afianzar el desarrollo de las habilidades motrices básicas.

- La actividad física realizada mediante juegos lúdicos ayuda al desarrollo motor de los niños, la motricidad gruesa favorece la motricidad y manejo corporal, es así, que el desarrollo motor se debe empezar en los primeros años de vida de los infantes, su proceso de crecimiento y maduración debe ser desarrollado de forma adecuada durante toda su etapa infantil.

- Las actividades motrices de carácter lúdico y recreativo mostraron un gran impacto en cuanto al desarrollo de la motricidad gruesa de los niños de cinco a siete años, 
beneficiando de tal manera el aprendizaje motriz, lo cual refuerza la evolución general de su personalidad e independencia.

- Las entidades educativas de educación inicial son las llamadas a generar cambios armoniosos en la motricidad gruesa de los infantes, pues las destrezas que el niño va obteniendo le permite mover armoniosamente su cuerpo, mantener el equilibrio y la coordinación; los docentes de educación corporal y maestros de educación física son los encargados del desarrollo armónico del niños mediante el apoyo de programas en educación motriz.

- Finalmente se puede mencionar que nuestro estudio luego del programa de intervención obtuvo puntuaciones significativas en siete de las ocho variables estudiadas; con lo cual en estudios futuros se debe dar mayor énfasis al desarrollo del equilibrio.

\section{Agradecimientos.}

Queremos expresar nuestro agradecimiento a la Universidad Técnica de Ambato, Facultad de Ciencias Humanas y Educación, Carrera de Cultura Física, y Pedagogía de la Actividad Física y Deporte por el apoyo para la realización de nuestro trabajo de investigación.

\section{Referencias bibliográficas.}

Andradas, A. E. (2015). Actividad física para la salud y reduccion del sedentarismo. MADRID.

Bayas, M. F. (2017). Actividades Lúdicas "Aprendo Jugando" Para Desarrollar La Motricidad Gruesa En Los Niños Y Niñas Del Primer Año De Educación Básica. European Scientific Journal, 13(26).

Benjumea, J. M. (2017). (Test de coordinación motriz 3JS: Cómo valorar y analizar su ejecución . Retos, 32, 189-193.

Bravo, I. R.-N. (2017). Diferencias en función del género en la puntería y atrape en niños de Educación Primaria. RETOS. Nuevas Tendencias en Educación Física, Deporte y Recreación, sin volumen (32), 35-38.

Cano-CappellacciI, M. L. (2015). Confiabilidad y validez de contenido de test de desarrollo motor grueso en niños chilenos. Rev Saúde Pública, 49, 97. doi:10.1590/S00348910.2015049005724

Carrillo, C. M. (2017). Actividad física e IMC de los universitrios de Veracruz y Colima. . Revista Mexicana de Investigación en Cultura Física y Deporte,, 53. 
Da Silva Carmo, C. G. (2015). Motricidad escolar: Reflexiones y acciones en una experiencia situada en la educación básica. ),. Estudios pedagógicos (Valdivia), 41, $51-65$.

Ferradas Garcia, C. (2015). Evaluación de la Lateralidad mediante el test de Harris en niños de 3 y 6 años. Universidad de Valladolid. Escuela Universitaria de Educación (Soria), 21-24.

Ferrandis, I. G. (2017). Estrategias didácticas innovadoras en la enseñanza de las ciencias. Enseñanza de las ciencias: revista de investigación y experiencias didácticas, 35(2), 109-126.

González, M. A. (2017). Análisis de la formación del maestro/a de educación infantil en expresión corporal. EmásF: revista digital de educación física(49), 36-59.

González, N. F. (2018). Actividad física y ejercicio en la mujer. . Revista Colombiana de Cardiología.

Henderson, Sheila E. D. A. (2016). Evaluación de la batería de evaluación del movimiento para niños-2 (MABC-2). EFPA, 3.

Hollund, I. M. (2018). White matter alterations and their associations with motor function in young adults born preterm with very low birth weight. NeuroImage: Clinical(17), 241-250.

León, B. M. (2015). Desarrollo motor de los niños indígenas atendidos por desnutrición en Valledupar. Nutr. clín. diet. hosp, 36(3), 76-78. Obtenido de http://revista.nutricion.org/PDF/maureth.pdf

Loza, S. B. (2017). La función del juego en el desarrollo integral de los niños de 2 a 4 años. . Revista PUCE.

Márquez, S. (1998). Analisis de la lateralidad y la eficiencia manual en un grupo de niños de 5 a 10 años. European Journal of Human Movement, (4), 131-139.

Martínez, F. D. (2017). Autoconcepto, práctica de actividad física y respuesta social en adolescentes: Relaciones con el rendimiento académico. . Revista Iberoamericana de Educación, 73.

Otalvaro, A. M. (2016). Instrumentos de evaluación de pesquisa de neurodesarrollo en la intervención temprana. tesis psicológica, 54 - 71.

Pérez, L. M. (2017). Evaluar la Coordinación Motriz Global en Educación Secundaria:El Test Motor SportComp. RICYDE. Revista Internacional de Ciencias del Deporte, XIII(49), 285-301 . 
Pérez, L. M. R., Moro, M. I. B., Otero, I. R., Nieto, M. P., Collado, N. R., Coll, M. V. G., \& Manzano, J. A. N. (2017). Evaluar la Coordinación Motriz Global en Educación Secundaria: El Test Motor SportComp. RICYDE. Revista Internacional de Ciencias del Deporte, 13(49), 285-301.

Pumar Vidal, B. N. (2015). Efectos de un programa de actividad física en escolares. Educación Física y Ciencia, 17(2), 1-13.

Rodríguez, M. G.-A.-M. (2017). La educación psicomotriz en su contribución al desarrollo del lenguaje en niños que presentan necesidades específicas de apoyo educativo. Revista de Investigación en Logopedia, 7(1), 89-106. Obtenido de http://www.redalyc.org/html/3508/350851047005/

Santana, D. O. (2016). Desarrollo motor grueso y estado nutricional en niños preescolares con presencia y ausencia de transición I, que cursan el nivel de transición II. Revista Ciencias de la Actividad Física, 18(1). Obtenido de https://scholar.google.es/scholar?hl=es\&as_sdt=0\%2C5\&as_ylo=2016\&q=motricid $\mathrm{ad}+$ gruesa+en+ni\%C3\%B1os\&oq=motricidad+gruesa

Sumner, E. L. (2018). Comparing attention to socially-relevant stimuli in autism spectrum disorder and developmental coordination disorder. . Journal of abnormal child psychology, 1-13.

Vallejos, M. S. (2016). Estudio comparativo entre el desarrollo psicomotor y el estado nutricional en niños de kínder, pertenecientes a un establecimiento municipal ya uno particular de la ciudad de Temuco. Revista Ciencias de la Actividad Física, 18(2). Obtenido de https://revistacaf.com/ojs/index.php/RCAF/article/view/82

Weisstaub, G. S. (2017). Lactancia materna, desarrollo motor y obesidad, $i$ Existe asociación causal? Revista chilena de pediatría, 88(4), 88(4), 451-457. doi:http://dx.doi.org/10.4067/S0370-41062017000400002

Zuluaga, A. E. (2015). MOTRICIDAD Y ENSEÑANZA: SABERES QUE TRANSITAN EN EL AULA. Revista Latinoamericana de Estudios Educativos, 11(2).

\section{【Ligncia}




\section{Para citar el artículo indexado.}

Mocha J., Ortiz P., Zapata E. \& Cárdenas A. (2018). Efectos de un programa de intervención en la motricidad gruesa: estudio con niños de 5 a 7 años. Revista electrónica Ciencia Digital 2(2), 64-78. Recuperado desde:

http://cienciadigital.org/revistacienciadigital2/index.php/CienciaDigital/article/view/73/68

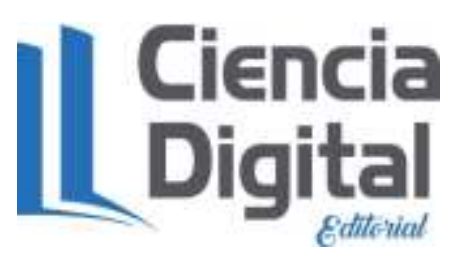

El artículo que se publica es de exclusiva responsabilidad de los autores y no necesariamente reflejan el pensamiento de la Revista Ciencia Digital.

El articulo queda en propiedad de la revista y, por tanto, su publicación parcial y/o total en otro medio tiene que ser autorizado por el director de la Revista Ciencia Digital.
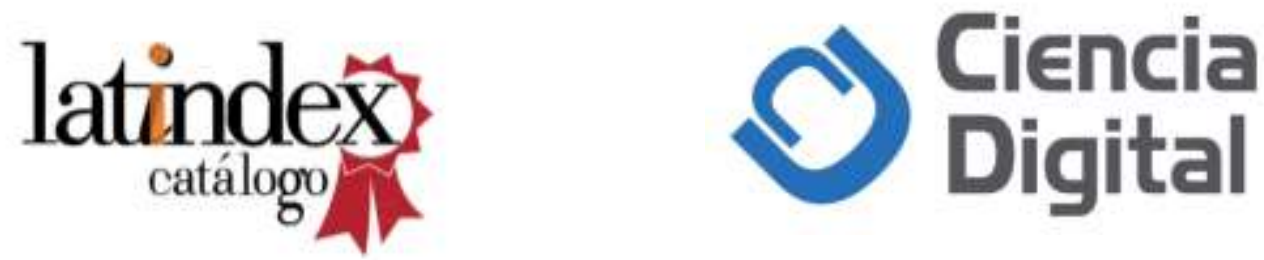